\title{
Optical determination of interface roughness in multilayered semiconductor structures
}

\author{
M.Mazilu', V.Donchev', A.Miller', O.Blum² \\ REPER \\ ${ }^{1}$ School of Physics and Astronomy, University of St.Andrews, \\ AUs 2 is 899 \\ North Haugh, St.Andrews, Fife KY16 9SS, Scotland \\ Fax: ++44-1334-46.31.04; e-mail:a.miller@st-andrews.ac.uk \\ OSTI \\ ${ }^{2}$ Sandia National Laboratories, MS0603, Albuquerque, NM 87185, USA
}

\begin{abstract}
$\underline{\text { Abstract }}$
We have developed a new approach (the LQR method) for calculating the reflectivity and transmission spectra of a multilayer optical material with $\mathrm{N}$ interfaces, as an altemative to the matrix method. The approach allows the inclusion of the effects of interface roughness by introducing a "rough" element between adjacent layers. For this purpose we have developed an empirical model, which describes the effect of interface roughness on an optical beam passing through or being reflected from an interface. An assessment of the interface roughness of a multilayer structure was carried out by fitting the experimental reflectivity spectrum of GaAs/AlGaAs multiple quantum well samples with and without oxidation of the barrier layers. The refractive index and the thickness of the oxidised layers were also obtained from the fit.
\end{abstract}

PACS: 81.05.Ea, 68.35.Ct, 78.20.Bh

The interface between different materials in multilayer semiconductor structures has received considerable attention recently. The interface roughness in such structures is an important parameter limiting both the optical and the electrical performance of devices [1-6]. 


\section{DISCLAIMER}

This report was prepared as an account of work sponsored by an agency of the United States Government. Neither the United States Government nor any agency thereof, nor any of their employees, make any warranty, express or implied, or assumes any legal liability or responsibility for the accuracy, completeness, or usefulness of any information, apparatus, product, or process disclosed, or represents that its use would not infringe privately owned rights. Reference herein to any specific commercial product, process, or service by trade name, trademark, manufacturer, or otherwise does not necessarily constitute or imply its endorsement, recommendation, or favoring by the United States Government or any agency thereof. The views and opinions of authors expressed herein do not necessarily state or reflect those of the United States Government or any agency thereof. 


\section{DISCLAIMER}

Portions of this document may be illegible in electronic image products. Images are produced from the best available original document. 
In particular, this problem is crucial to selectively oxidised AlAs/AlGaAs multilayers, which are now becoming widely used to form high reflectivity and wide-bandgap distributed Bragg reflectors (DBRs) in vertical cavity optoelectronics devices, such as lasers, LEDs, modulators and detectors. The scattering loss by interface roughness decreases the maximum reflectivity of DBRs, thus deteriorating the device performance.

Several authors have studied the $\mathrm{Al}$ (oxide) layers and the interface quality of $\mathrm{Al}$ (oxide)/AlGaAs structures [6-11]. Transmission electron microscopy (TEM) has revealed a granular structure of the $\mathrm{Al}$ (oxide) $[7,8]$ and the grain size has been estimated to be about $8 \mathrm{~nm}$ [8]. A porous interface between $\mathrm{Al}$ (oxide) and $\mathrm{AlGaAs}$ has been observed $[8,9]$ and an interface transition width of $2 \mathrm{~nm}$ has been reported in [7].

The reflectance method is a simple, non-destructive and direct way to study the optical properties of selectively oxidised AlAs/AlGaAs multilayers $[6,10,11]$. In this work we measure the reflectivity spectra of GaAs/AlGaAs multiple quantum well structures with and without oxidation of the barrier layers. A new approach is developed for calculating the reflectivity and transmission spectra of a multilayer optical structure with $\mathrm{N}$ interfaces. It includes a simple statistical model to describe the effect of interface roughness. By fitting the experimental reflectivity spectrum using this model we have assessed the interface roughness of the oxidised layers, as well as the $\mathrm{Al}$ (oxide) parameters (refractive index and thickness).

\section{Experimental}

The samples are grown by molecular beam epitaxy on (100) semi-insulating GaAs substrates with $2^{\circ}$ misorientation towards (110). A GaAs (400 nm) buffer layer is followed by 35 periods with each period containing an $\mathrm{Al}_{0.98} \mathrm{Ga}_{0.02} \mathrm{As}(50 \mathrm{~nm})$ barrier layer, an $\mathrm{Al}_{0.4} \mathrm{Ga}_{0.6} \mathrm{As}(7.5 \mathrm{~nm})$ spacer, a GaAs $(7 \mathrm{~nm})$ quantum well and another $\mathrm{Al}_{0.4} \mathrm{Ga}_{0.6} \mathrm{As}(7.5 \mathrm{~nm})$ spacer. $\mathrm{An} \mathrm{Al}_{0.98} \mathrm{Ga}_{0.02} \mathrm{As}(50 \mathrm{~nm})$ barrier layer and a $\mathrm{GaAs}(30 \mathrm{~nm})$ cap layer completed the 
structure. Three samples were used. The first (2408uu) is as-grown. The other two (2408e and 2408c) were patterned and etched (RIE, $36 \mathrm{~min}$ ) to obtain $20 \mu \mathrm{m}$ stripes spaced by $2 \mu \mathrm{m}$ grooves. The $\mathrm{Al}_{0.98} \mathrm{Ga}_{0.02} \mathrm{As}$ layers in sample $2408 \mathrm{c}$ have been laterally oxidised at $398^{\circ} \mathrm{C}$ for 70 min using a flow of ( ?? ) gas bubbled through $\mathrm{H}_{2} \mathrm{O}$ heated to (?? ${ }^{\circ} \mathrm{C} .$. Scanning electron microscopy has shown that these conditions ensure a complete oxidation of the $\mathrm{Al}_{0.98} \mathrm{Ga}_{0.02} \mathrm{As}$ layers.

In our set-up for reflectivity measurements the collimated beam from the light source (a tungsten lamp with a stabilised power supply) is split into two beams, which are reflected from the sample and from a reference mirror, respectively. The beams are recombined and focused onto the entrance slit of a monochromator, coupled to a Si photodetector. We have employed an original technique for simultaneous recording the two optical beams using a single optical chopper, detector and lock-in amplifier [12]. It has the advantages of real time spectral corrections and noise reduction. The new feature of our method is based on chopping the two beams with the same frequency but with a phase shift of $\pi / 2$ or $3 \pi / 2$. The corresponding electrical signals from the detector are measured as the in-phase and the quadrature signal by the lock-in amplifier and their ratio gives the relative reflectivity of the sample with respect to the reference mirror.

\section{LQR method}

The reflectivity and transmission of multilayer structures are usually calculated by the transfer matrix method [13] or its modification $[6,14]$. An alternative way is the recurrence formulae, which have been employed in X-ray diffraction studies $[15,16]$. In the case of periodic structures both approaches lead to Chebyshev polynomials of the second kind $[13,15]$. 
In this paper we propose a new method which has been developed here for normal incidence of light, but can be easily generalised for oblique incidence. It is based on the recurrence formulae, but is easier to calculate and more straightforward in comparison with the calculations used in $\mathrm{X}$-ray studies $[15,16]$. We also take into account also the changes in the phase and the amplitude of the wave during its propagation in each layer.

The starting point is the fact that the reflectivity and transmission of a multilayer structure do not change if the layers are separated by infinitely narrow vacuum gaps [16]. Thus, the layers of the original structure become independent of each other and can be characterised by their field reflection $L, R$ and transmission $T$ coefficients with respect to vacuum. Here $L$ and $R$ stand for left and right travelling wave, respectively. For each layer or set of adjacent layers we define consistently a vector $X$ with three components, as follows: $X(1)=L ; X(2)=T^{2}-L R \equiv Q ; X(3)=R$. We use the combination $Q=T^{2}-L R$ instead of the transmission $T$, because this way the calculations simplify and the representation of the results becomes more concise and straightforward. The components of $X$ for a single layer can be easily calculated.

$$
L=R=\left(r-r p^{2}\right) /\left(1-r^{2} p^{2}\right)
$$

$$
Q=\left(p^{2}-r^{2}\right) /\left(1-r^{2} p^{2}\right)
$$

Here $r=(1-n) /(1+n)$ is the field reflection coefficient of the interface vacuum/material, $n$ is the complex refractive index, $\lambda$ is the wavelength, $d$ is the layer thickness, $p=\exp \left(i k_{0} n d\right)$ is the propagation factor and $k_{0}=2 \pi \lambda$. In the case of two layers separated by an infinitely narrow vacuum, the components of the vector $X$ can be found by applying the recurrence formulae for $L, R$ and $T$ and the definition of $X$. They are

$$
L=\left(L_{1}+L_{2} Q_{1}\right) /\left(1-R_{1} L_{2}\right)
$$




$$
Q=\left(Q_{1} Q_{2}-L_{l} R_{2}\right) /\left(1-R_{1} L_{2}\right)
$$

$$
R=\left(R_{2}+R_{1} Q_{2}\right) /\left(1-R_{l} L_{2}\right)
$$

Here the indices 1 and 2 refer to the first and second layers, respectively. The equations (2) can be applied to any two adjacent parts of the structure, each one containing one or several layers. We denote the addition of two adjacent elements of the structure with vectors $X_{1}=\left\{L_{1}, Q_{1}, R_{1}\right\}$ and $X_{2}=\left\{L_{2}, Q_{2}, R_{2}\right\}$ by $X_{X}=X_{1} \odot X_{2}$, where the sign (๑) corresponds to equations (2). It should be emphasized that the operation (c) is associative, but not commutative. Based on this operation we have constructed a specific algebra of the vectors $X$. For exemple the null element in this algebra is the vector $N=\{0,1,0\}$. Indeed according to (2)

$X \odot N=N \odot X=X$. Using (1) and the operation $\odot$ we can construct the whole structure under consideration and determine its reflectivity and transmission provided that the refractive indices and the thicknesses of the layers are known. We call our approach the LQR method.

A single interface, as well as the distance $d$ between the interfaces of a layer can also be characterized by $X$ vectors. For the interface vacuum/material $X=\{r, 1,-r\}$ (note that for one interface $t_{12} t_{21}-r_{12} r_{21}=1$ ), while the vector corresponding to the propagation between the two interfaces is $\left\{0, p^{2}, 0\right\}$. It is easy to check that the $X$ vector of a layer in vacuum (equations (1)) is obtained from $\{r, 1,-r\} \odot\left\{0, p^{2}, 0\right\} \odot\{-r, 1, r\}$.

\section{Model for the interface roughness}

Several models for rough surfaces and interfaces could be found in the literature $[1,4-$ $6,14-18]$, but only few works have applied such models to analyse optical reflectivity data $[6,14]$. In this study we apply a statistical treatment of the interaction of an optical beam with a rough interface. It is similar to that proposed by Davies [17] for scattering of radar waves 
from rough water surfaces. In a first approximation the interface is represented as an ensemble of parallel steps, neglecting the effects of different step slopes. The depths of the steps are statistically distributed with a distribution function $s(z)$, where $z$ is the growth direction. This distribution is normalised and its mean value, which corresponds to the position of the ideally flat interface, is chosen for the origin of the $z$ - axis. The standard deviation of $s(z)$ is a measure of the roughness. When a coherent monochromatic plane wave is reflected from or transmitted through a rough interface it becomes a mixture of several parts; which have followed different optical paths and therefore have different phases and amplitudes. The wave loses its coherence and acquires a phase distribution.

To include the interface roughness effects in the reflectivity calculations we introduce a "rough" element between adjacent layers. It is formed by the materials of the two layers separated by an internal rough interface, represented as explained above. However its outside interfaces are flat and it is spaced by infinitely narrow vacuum gaps from the normal layers. Consider a step in the rough interface between materials 1 and 2 at a depth $z$. In the framework of the LQR algebra the $X$ vector of the "rough" element at this point is given by

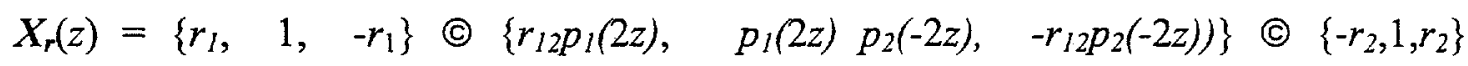

Here $r_{\mathrm{j}}$ are the reflection coefficients and $p_{\mathrm{j}}(z)=\exp \left(i k_{0} n_{\mathrm{j}} z\right)(\mathrm{j}=1,2)$ are the propagation factors of the two materials. The total reflected or transmitted field is given by the interference of the beams reflected from or transmitted through steps with different $z$. This is taken into account by integrating the "rough" vectors $X_{r}(z)$ weighted with the distribution function $s(z)$. Note that only the middle part of $X_{r}(z)$ depends on $z$. The function $s(z)$ is usually assumed to be Gaussian $\left(s(z)=\left(2 \pi \sigma^{2}\right)^{-1 / 2} \exp \left(-z^{2} / 2 \sigma^{2}\right)\right)[6,14,17]$. In this case one obtains : 


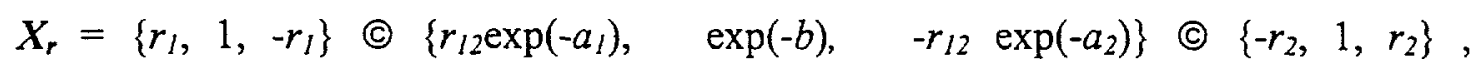

where $a_{l}=2\left(\sigma k_{0} n_{l}\right)^{2}, a_{2}=2\left(\sigma k_{0} n_{2}\right)^{2}, b=2\left[\sigma k_{0}\left(n_{l}-n_{2}\right)\right]^{2}$ and $\sigma$ is the standard deviation of the Gaussian $s(z)$. So, the "rough" element between two layers depends on their refractive indices, the interface roughness and the wavelength. Its effect is more pronounced for larger index difference and larger $\sigma \lambda$. It is easy to check that if one of the conditions $\sigma=0$ or $n_{1}=n_{2}$ is fulfilled $X_{r}=N$ and therefore the "rough" element does not affect the calculated results.

\section{Results and Discussion}

In the calculations we have used the Afromovitz model [19] for the refractive index of GaAs and $\mathrm{Al}_{0.4} \mathrm{Ga}_{0.6} \mathrm{As}$, while for $\mathrm{Al}_{0.98} \mathrm{Ga}_{0.02} \mathrm{As}$ the model of Fern and Onton [20] has been applied. No excitonic effects have been considered. The reflectivity spectrum of the as-grown sample (2408uu) is shown in Fig.1. A series of interference fringes are observed with a period of $30-40 \mathrm{~nm}$, which increases with wavelength as expected. In order to achieve optimal matching in the simulation we have assumed a small deviation of the refractive indices over the whole spectral range considered. This is $+1.5 \%$ for $\mathrm{GaAs}$ and $\mathrm{Al}_{0.4} \mathrm{Ga}_{0.6} \mathrm{As}$ and $+0.3 \%$ for $\mathrm{Al}_{0.98} \mathrm{Ga}_{0.02} \mathrm{As}$. We fix these values in the analysis of the other samples. No roughness has been considered in this case. For multilayers with close refractive indices such as in the GaAs/AlGaAs system, the effect of the roughness in our model becomes noticeable for $\sigma>4$ $5 \mathrm{~nm}$.

When simulating the reflectivity of a single layer with the thickness of whole the structure (2600 $\mathrm{nm}$ without the buffer layer) and a refractive index averaged in proportion to the thicknesses of the different materials we obtain fringes which are shifted along the $\mathrm{Y}$-axis, but have the same wavelength positions. This means that the observed fringe pattern is 
determined predominantly by the interference of the light reflected from the front surface and from the last interface $\left(\mathrm{Al}_{0.98} \mathrm{Ga}_{0.02} \mathrm{As} / \mathrm{GaAs}\right)$.

In the case of etched samples the contribution of the layers at the bottom of the grooves has been added into the simulations in proportion to their area. Unlike the work of MacDougal [10] we have not been able to fit the experimental curves without considering these layers. Their effect is revealed as a modulation of whole the spectrum with a period of about $100 \mathrm{~nm}$, which corresponds to a groove depth $d_{g r}$. The extent of the modulation depends on the roughness $\sigma_{g r}$ of their surfaces. The reflectivity spectrum of the etched, but nonoxidised sample (2408e) is displayed in Fig.2. The fringes are at the same positions as for the as-grown sample, but their amplitudes and the mean reflectivity value are modulated, because of the groove layers. Optimal fitting is obtained for $d_{g r}=3335 \mathrm{~nm}$ and $\sigma_{g r}=80 \mathrm{~nm}$.

The oxidation of AlGaAs layers changes considerably their parameters [7-11]. Shrinkage of layer thickness is commonly observed. TEM [7,9] and reflectance [11] studies have found different values for the extent of this shrinkage, which range between $3 \%$ and $13 \%$. The refractive index decreases by approximately a factor of 2 . Values in the range $1.5-$ 1.65 have been obtained by ellipsometric and reflectance measurements $[10,11]$. It is usually assumed to be wavelength independent in the visible and the near-infrared regions.

Figure 3 represents the reflectivity spectrum of the oxidised sample (2408c). In comparison with the two previous cases the period of the fringes is increased by about 1.5 times, which corresponds to the decrease of the effective refractive index and thickness of the structure after oxidation. Besides, the fringe amplitude is smaller and the mean reflectivity value is lower. This is attributed to light scattering by interface roughness of the oxidised layers. The modulation of the spectrum due to the groove layers has a similar extent as in Fig.2, but is more clearly observed, because of the smaller fringe amplitude. 
In the simulations we have varied the index and the thickness of the oxidised layers and the roughness of their interfaces. An optimal fit is obtained with an oxide index of 1.54, oxide thickness of $44.5 \mathrm{~nm}$, or $89 \%$ of the initial value and a roughness of $8.4 \mathrm{~nm}$. The groove parameters in this case are slightly different: $d_{g r}=3247 \mathrm{~nm}$ and $\sigma_{g r}=70 \mathrm{~nm}$, which can be explained by changes due to the oxidation. The obtained values for the refractive index and the shrinkage extent are in good accordance with the findings of other authors [7,9-11]. The interface roughness of $\mathrm{Al}$ (oxide) layers is larger that the result obtained in [6], but corresponds to the reported grain size of the oxide granular structure [8].

\section{Conclusion}

The LQR method proposed in this paper is a new approach for calculating the reflectivity and transmission spectra of a multilayer structure with $\mathrm{N}$ interfaces. With its straightforward nature and simplicity for computing it could be an useful alternative to the matrix method. The effects of interface roughness can be easily included in the LQR calculations by introducing a "rough" element between adjacent layers. For that purpose we have developed a simple statistical model of the rough interface. The model is applied to GaAs/AlGaAs multiple quantum well structures with and without oxidation of the barrier layers. The fit to the reflectivity spectra have given important parameters for the oxidised layers such as the interface roughness, the refractive index and the thickness. Similar analysis can be carried out in the case of a wide range of multilayer materials. The developed theory can be applied not only to optical radiation but also to any electromagnetic wave.

Acknowledgements. One of the authors (V.D.) acknowledges NATO, the Royal Society and the British Foreign and Commonwealth Office for his post-doctoral fellowship. EPSRC and NATO collaborative grants are also gratefully acknowledged.

Sandia is a multiprogram laboratory operated by Sandia Corporation, a Lockhed Martin Company, for the United States Departmen of Energy under contract DE-ACO4-94AL85000. 


\section{References}

1. F. Yang, M.Wilkinson, E.J. Austin, K.P. O’Donnell: Phys.Rev.Lett. 70, 323 (1993)

2. Y.-G. Zhao, Y.-H. Zou, Y.-D. Qin, X.-L. Nuang: Appl. Phys. Lett. 72, 97 (1998)

3. W. Braun, L.V. Kulik, T. Baars, M. Bayer, A. Forchel: Phys.Rev.B 57, 7196 (1998)

4. T.G. Dargam, B. Koiller: Solid State Commun. 105, 211 (1998)

5. Y. Fu, Y.-M. Mu, M. Willander: Superlattices and Microstr. 23, 417 (1998)

6. H.-E. Shin, Y.-G. Ju, H.-W. Song, D.-S. Song, I.-Y. Han, J.-H. Ser, H.-Y. Ryu, Y.-H. Lee: Appl. Phys. Lett: 72, 2205 (1998)

7. T. Takamori, K. Takamesa, T. Kamijoh: Appl. Phys. Lett 69, 659 (1996)

8. S. Guha, F. Agahi, B. Pezeshki, J.A. Kash, D.W. Kisker, N.A. Bojarczuk: Appl. Phys. Lett 68, $906(1996)$

9. Z. Liliental-Weber, M. Li, G.S. Li, C.Chang-Hasnain, E.R. Weber: Proc. Microscopy and Microanalysis. (Eds. G.W.Bailey, J.M.Corbett, R.V.W.Dimlich, J.R.Michael and N.J.Zaluzec), San Francisco Press, San Francisco, 1996, p.942

10. M.H. MacDougal, H. Zhao, p.D. Dapkus, M. Ziari, W.H. Steier: Electronics Lett. 30, $1147(1994)$

11. P. Heremans, M. Kuijk, R. Windisch, J. Vanderhaegen, H.De Neve, R. Vounckx, G. Borghs: J.Appl.Phys. 82, 5265 (1997)

12. V. Donchev, M. Mazilu, A.Miller : paper presented at the conference LEOS'98, 1-4 December 1998, .Orlando, USA

13. M.Born, E.Wolf . Principles of Optics, $\left(6^{\text {th }}\right.$ corrected edition). Oxford, Pergamon Press 1983

14. J.Faist, J.-D. Ganiere, Ph. Buffat, S. Sampson, F.-K. Reinhart: J.Apl.Phys. 66, 1023 
(1989)

15. Y.N. Belyaev, A.V. Kolpakov: Phys.Stat.Sol (a) 76, 641 (1983)

16. A.V. Andreev, J.R. Prudnikov: Crystallogr. Rep. 41, 203 (1996). Translated from Kristallografyia 41, $220(1996)$

17. H.Davies: Proc. Inst. Elec. Engrs. 101, 209 (1954)

18. T. Yamaguchi, M.Nasu, Zh.-T. Jiang, M. Tabe, Y. Kanda: Thin Solid Films, 313-314, 264 (1998)

19. M.A. Afromovitz: Solid State Commun. 15, 59 (1974)

20. R.E. Fern, A. Onton: J.Appl.Phys. 42, 3499 (1971) 


\section{Figure Captions}

Fig.1. Measured and calculated reflectivity spectrum of the as-grown GaAs/AlGaAs multiple quantum well sample

Fig.2. Measured and calculated reflectivity spectrum of the GaAs/AlGaAs multiple quantum well sample which has been patterned, but not oxidised.

Fig.3. Measured and calculated reflectivity spectrum of the GaAs/AlGaAs multiple quantum well sample, which has been patterned and oxidised. 


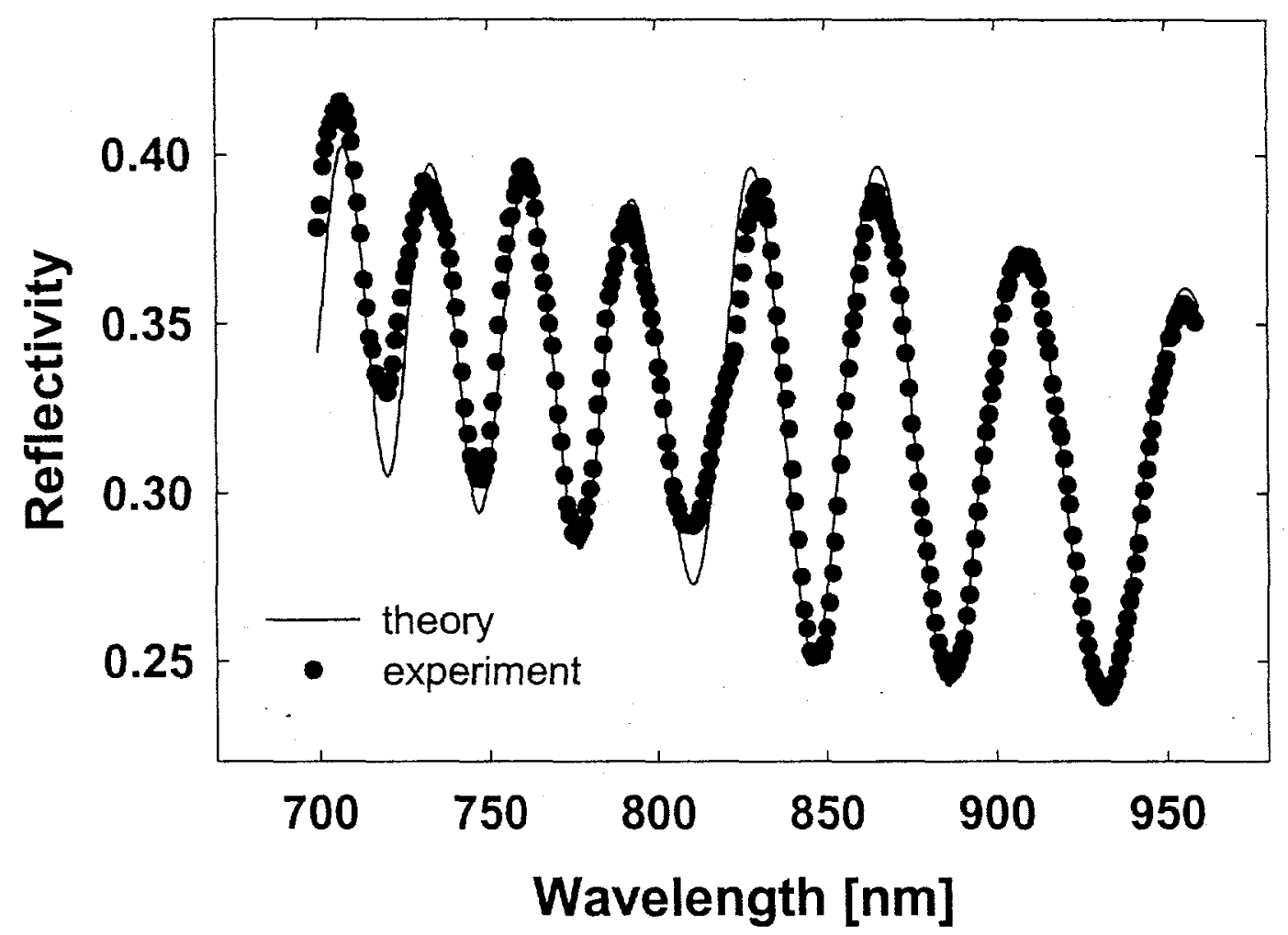

Fig.1 


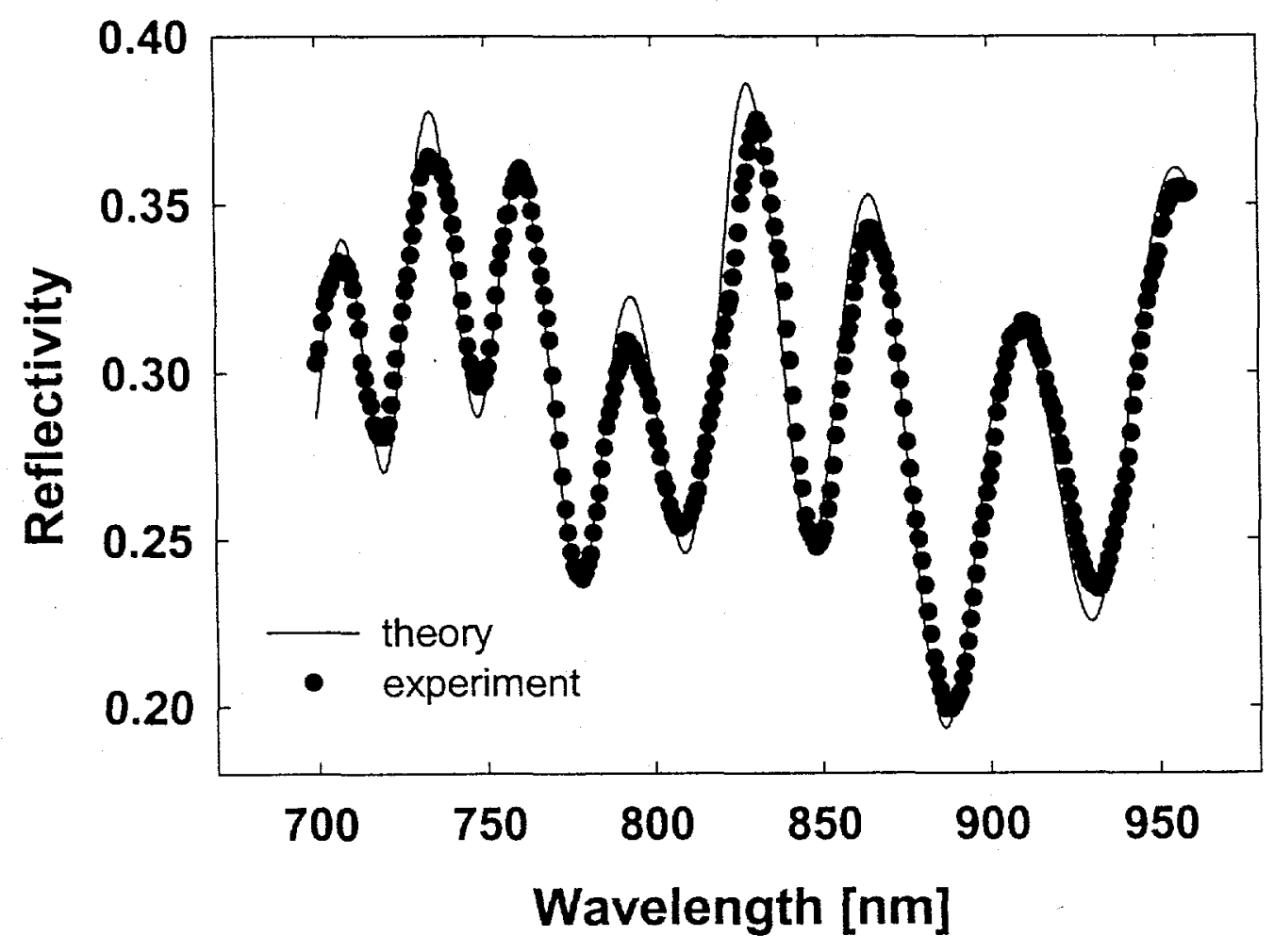

Fig.2 


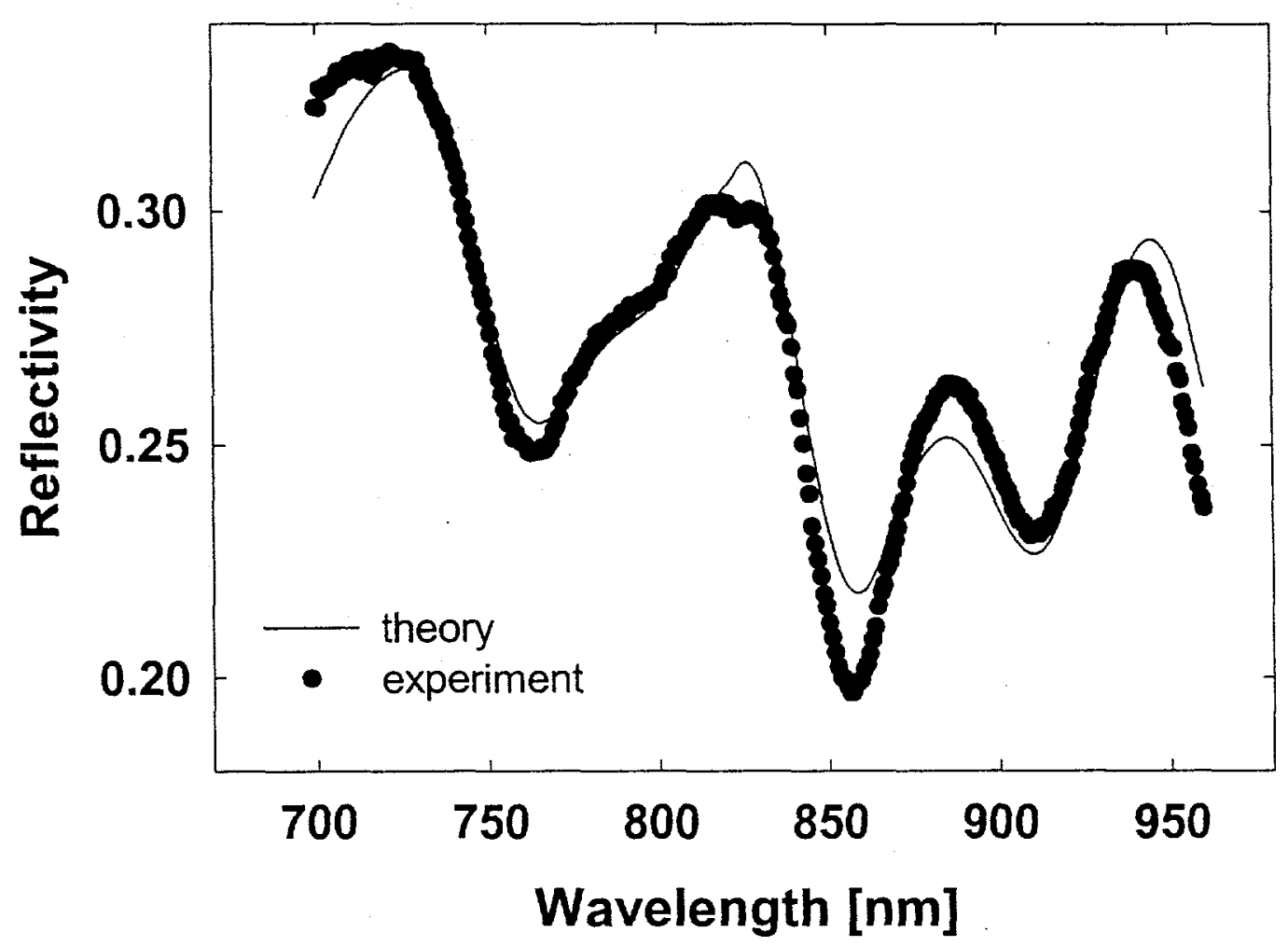

Fig.3 